\title{
Les cartes conceptuelles : un outil au service de l'évaluation des compétences
}

\author{
Concept maps: a competency assessment tool
}

\author{
Pascale CHASTRAGNAT ${ }^{1,2}$ et Claire MARCHAND ${ }^{2}$ \\ 1 Institut de formation aux métiers de la santé, Sens, France \\ 2 Laboratoire de pédagogie de la santé, UPRES 3412, Université Paris 13 Bobigny, Sorbonne Paris Cité, France \\ Manuscrit soumis à la rédaction le 16 décembre 2011 ; commentaires éditoriaux formulés aux auteurs le 29 mai \\ et le 15 juillet 2012 ; accepté pour publication le 3 septembre 2012
}

\begin{abstract}
Mots-clés
formation en soins

infirmiers ;

évaluation formative ;

compétence ;

ressources ; carte

conceptuelle

\section{Keywords}

nursing training;

formative assessment;

competence;

resources; concept

map

Résumé - Contexte : Le nouveau référentiel de formation, conduisant au diplôme d'État d'infirmier en France, s'inscrit dans une logique de compétence. L'évaluation de la compétence, concept abstrait et polymorphe, relève du défi et nécessite de trouver de nouveaux modes d'évaluation. La carte conceptuelle pourrait en être un. Objectif : Le but de l'étude est d'explorer l'intérêt de la carte conceptuelle pour l'évaluation d'une compétence en soins infirmiers. Il s'agira de repérer l'identification des ressources mobilisées par les étudiants et leur niveau d'organisation pour traiter une situation. Méthodes : Des étudiants en soins infirmiers de deuxième année ont réalisé une carte conceptuelle présentant les ressources pour déployer une compétence du référentiel en situation. Les cartes ont été analysées sur la base d'une carte de référence élaborée par trois experts et des données de la littérature. Résultats : 20 cartes conceptuelles ont été analysées. Les ressources internes (RI) sont plus souvent intégrées que les ressources externes (RE). 10 étudiants identifient entre 20 et $30 \%$ des RI proposées dans la carte de référence. Pour 16 étudiants, moins de $10 \%$ des RE sont identifiées. Tous les étudiants font des propositions de ressources complémentaires à celles de la carte de référence. Conclusion : La carte conceptuelle permet d'évaluer les ressources mobilisées dans le développement d'une compétence. Associée à une étape d'explicitation et de rétro information, la carte conceptuelle procure à l'étudiant un feed-back intéressant sur son apprentissage et au formateur des pistes pour son enseignement.

Abstract - Background: Since the implementation of the new nursing training curriculum in France, teaching methods have focused on integrating student knowledge into a competency framework. However, competence remains an abstract concept that is difficult to define and accordingly to assess. New assessment tools are needed and concept maps might be part of them. Objective: The goal of the study is to explore the value of concept maps in assessing competence in the nursing profession. The key focus will be to establish to what extent the creation of such maps enable us to identify the various resources used by students and their level of organization in terms of dealing with a given situation. Methods: Second-year nursing students developed a concept map showing the resources needed in deploying one competency. The students' maps were analyzed in relation to a reference concept map based on one created by three experts as well as data from the literature. Results: 20 concept maps
\end{abstract}


were analyzed. Findings showed that internal resources (IR) were more often integrated $(n=20)$ than external resources (ER) $(n=17) .10$ students identified between 20 and $30 \%$ of the IR featured in the reference concept map. Most students (16) identified less than $10 \%$ of ERs. All students suggested resources, which were in keeping with those of the reference concept map. Conclusion: Concept maps help assess the resources used by students in the development of a competency. Associated with detailed explanations, concept maps provide students with interesting feedback on their learning, and teachers with useful information to improve their teaching.

\section{Introduction}

La réforme du référentiel de formation conduisant au diplôme d'État d'infirmier en France (arrêté du 31 juillet 2009) s'inscrit dans une nécessaire évolution du métier en raison de l'évolution même des techniques, des modes de prise en charge des patients en situation complexe, des attentes du public, ainsi que de l'évolution démographique des professionnels de santé. Cela implique désormais de former des professionnels ayant encore plus de capacités d'adaptation à un contexte en changement permanent. Ce nouveau référentiel se veut valorisant pour les infirmiers actuels et futurs et c'est une opportunité pour rentrer dans le système " licence-master-doctorat», dit LMD. Le référentiel décrit les activités du métier puis les compétences au nombre de dix (Annexe 1).

L'entraînement réflexif devient une exigence de la formation, permettant aux étudiants de comprendre la liaison entre savoirs et actions et donc d'intégrer les savoirs dans une logique de construction de la compétence. Les modalités pédagogiques doivent dès lors être orientées vers la construction par l'étudiant de ses connaissances à partir des savoirs. Elles relèvent donc d'une pédagogie différenciée qui met en évidence la diversité des fonctionnements individuels ${ }^{[1]}$.

Pour nombre de formateurs en institut de formation en soins infirmiers (IFSI), ces bouleversements ont généré des difficultés d'ordre pédagogique et organisationnel. Ils se sont trouvés démunis face à un changement d'approche pédagogique, qui visait à substituer les compétences aux connaissances en tant que finalités à atteindre.

Pour obtenir le diplôme dans sa totalité, l'étudiant doit être évalué et certifié sur les dix compétences postulées dans le référentiel. L'article 43 précise que : « chaque compétence s'obtient de façon cumulée : a) par la validation de la totalité des unités d'enseignement en relation avec la compétence ; b) par l'acquisition de l'ensemble des éléments de la compétence évalués lors des stages ; c) par la validation des actes, activités et techniques de soins évalués soit en stage, soit en institut de formation ». L'évaluation des compétences, que ce soit pour les formateurs ou pour les tuteurs de stage, relève du défi. En effet, il n'y a plus de mise en situation professionnelle soumise à évaluation critériée. Le portfolio, nouvellement instauré, permet d'évaluer certains aspects des compétences mais semble cependant être insuffisant. En stage, la performance est davantage jugée que les ressources mobilisées.

Il semble donc nécessaire de trouver de nouveaux modes d'évaluation dans la mise en œuvre des compétences. La carte conceptuelle pourrait en être un.

\section{Le concept de compétence}

Aujourd'hui, différents auteurs s'accordent sur une définition de la compétence avec quelques nuances de formulation $^{[2-4]}$. Nous retenons dans ce travail la définition de Tardif ${ }^{[2]}:$ « la compétence est un savoir-agir complexe qui prend appui sur la mobilisation et la combinaison efficaces d'une variété de ressources internes et externes à l'intérieur d'une famille de situations. Elle se développe tout au long de la vie». Pour éclairer cette définition, Scallon ${ }^{[3]}$ explicite ce que n'est pas la compétence, à savoir que : a) elle ne se réduit pas à un ensemble de résultats observables ; b) elle ne peut être confondue avec la performance, 
qui désigne davantage la manifestation de la compétence; c) elle doit se rapporter à une situation précise ; d ) elle ne se réduit pas à un corpus de connaissances ou de savoir-faire ; e) elle ne peut être confondue avec la notion d'habileté ; elle doit être différenciée de la notion d'objectif, bien que ce soit deux possibilités d'exprimer des attentes.

Cette définition considère donc une compétence comme un savoir-agir complexe qui la différencie ainsi d'un simple savoir-faire, d'une procédure ou d'une connaissance procédurale. Elle s'éloigne d'une logique comportementaliste ou béhavioriste pour adopter une logique plus systémique. Il est important de distinguer « savoir-faire en situation », qui correspondrait à l'exécution d'une opération prescrite et à l'application d'instructions, pouvant être circonscrit et stabilisé (automatisation possible), d'un « savoir-agir en situation » où l'on demande à l'individu d'être polyvalent, de prendre des initiatives, d'agir efficacement dans des situations où il ne dispose pas de toutes les informations nécessaires, qu'il est donc invraisemblable d'automatiser.

Selon Le Boterf ${ }^{[4]}$, pour être reconnu comme compétent il ne suffit plus seulement d'être capable d'exécuter le prescrit mais il faut aller au-delà du prescrit. Le savoir-agir suppose de savoir combiner et mobiliser des ressources appropriées et de savoir mettre en œuvre des pratiques professionnelles pertinentes. Ce savoir-agir est forcément complexe et contextualisé ${ }^{[2]}$.

La compétence serait la capacité à agir efficacement en mobilisant des ressources internes et des ressources externes en situation complexe et nouvelle. À ce jour, le terme de « ressources », entendu dans un sens très englobant, remplace celui de «connaissances » qui est plus limitatif. Il existe une multitude de ressources de natures variées qui intègrent tous les types de composantes de la compétence (cognitives, affectives, sociales, sensorimotrices), susceptibles d'être mobilisées en actions finalisées face à une famille de situation.

Les ressources internes comprennent : les connaissances déclaratives (correspondant essentiellement à des connaissances théoriques), les connaissances procédurales (correspondant au comment de l'action) et les connaissances conditionnelles (correspondant au quand et au pourquoi de l'action) ${ }^{[5]}$, les attitudes, les schèmes et les habitus acquis dans le domaine expérientiel ${ }^{[4]}$. Les ressources externes à l'individu peuvent se révéler aussi cruciales que les ressources internes. Il peut s'agir par exemple : d'un dictionnaire consulté dans une démarche d'écriture, des technologies de l'information et de la communication pour constituer une banque de données, d'un logiciel que l'on emploie pour traiter de l'information, des collègues que l'on interroge sur des pratiques, des tuteurs de stage qui participent à l'encadrement.

Certaines ressources vont devenir des savoir-faire automatisés ce qui permet à l'apprenant de se dégager de l'attention à leur porter. Cela participe du caractère intégrateur de la compétence. Mais il ne suffit pas de posséder des ressources, encore faut-il savoir les utiliser à bon escient dans des contextes particuliers ${ }^{[6]}$. La mobilisation et surtout la combinaison des ressources se situent au cœur de toute compétence et constituent son caractère combinatoire.

Enfin, selon la définition retenue, la compétence doit être contextualisée, définie par une famille de situation clairement circonscrite. Celle-ci est constituée d'un ensemble de situations de niveaux de difficulté et de complexité équivalentes qui sollicitent les mêmes compétences ${ }^{[7]}$. Elles sont parfois déclinées en situation cible, situation de réinvestissement, situation d'intégration. L'exercice de la compétence par l'apprenant se réalise donc par l'intermédiaire d'une nouvelle situation à l'intérieur d'une même famille.

Le caractère développemental de la compétence sous-tend que celle-ci sera déployée tout au long de la formation et son caractère évolutif se comprend par la possibilité que de nouvelles ressources internes et externes pourront y être associées, de façon non limitée, sans dénaturer la compétence.

À partir d'une définition commune de la compétence il devient possible d'envisager son évaluation.

\section{Comment évaluer une compétence ?}

Deux intentions évaluatives sont habituellement distinguées : l'évaluation formative et l'évaluation sommative (auteurs d'origine américaine) ou certificative 
(auteurs européens). L'évaluation formative (formative assessment) est définie comme un processus d'évaluation continue ayant pour objectif d'assurer la progression des individus engagés dans une démarche d'apprentissage. La décision qui fait suite à l'évaluation consiste en une régulation qui a pour objet, soit la situation d'apprentissage, soit l'individu luimême ${ }^{[3]}$. L'évaluation certificative (summative assessment) fait référence à une sanction de fin d'études ou à la reconnaissance d'un niveau de développement au terme d'une période donnée.

Le terme d'évaluation authentique (authentic assessment ${ }^{[2]}$ est de plus en plus utilisé, en particulier pour l'évaluation des compétences. Dans ce type d'évaluation, on insiste fortement sur l'idée que le contenu des situations d'évaluation doit correspondre à des problématiques de la « vraie vie », qui existent réellement en dehors de l'institution de formation. L'évaluation des compétences consisterait en un processus de collecte de données à partir de sources multiples et variées qui, en considérant les expériences d'apprentissage, permettrait de rendre compte du niveau de développement des compétences de l'apprenant, de son degré de maîtrise des ressources internes et externes pouvant être mobilisées dans leur mise en œuvre et de l'étendue des situations dans lesquelles l'apprenant est en mesure de déployer ces niveaux de développement et ces degrés de mâ̂trise $^{[2]}$.

Les auteurs s' accordent pour constater que la pratique de l'évaluation des compétences comporte beaucoup d'incertitudes et il semble en effet difficile de déterminer de façon exhaustive tout ce que sait une personne et tout ce qu' elle peut faire. Il serait possible alors d'envisager l'évaluation des compétences comme une vidéographie plutôt que comme une photographie car elle se situe dans ce que Tardif appelle une trajectoire de développement ${ }^{[2]}$.

Au moment de l'évaluation des compétences, il faudra se préoccuper de la capacité de l'étudiant à mobiliser ou à combiner les ressources dans des contextes précis mais aussi de celles qu'il aurait dû mobiliser. De plus, l'évaluateur doit estimer le degré de transférabilité des ressources de l'étudiant face à différentes problématiques. L'identification d'une famille de situations permet de situer le champ d'action d'une compétence donnée et ainsi les lieux possibles de transfert des apprentissages.

Ainsi, il importe de mettre en place des moyens ou des outils qui obligent les apprenants à exprimer ouvertement les ressources sur lesquelles ils prennent appui, pour que les formateurs aient accès aux mobilisations et aux combinaisons mises en œuvre dans le cadre du développement d'une compétence, charge à l'étudiant de faire des liens entre les tâches et les activités spécifiques accomplies, les connaissances construites et les compétences développées. Cependant, force est de constater la difficulté pour les apprenants à établir des liens entre toutes les ressources souvent fragmentées et à élaborer une pensée complexe $\mathrm{e}^{[8]}$.

On peut aider l'apprenant à faire des liens en lui proposant d'élaborer des arbres de connaissances, pratique remontant à des temps ancestraux (apprentissage de la médecine au Tibet depuis des millénaires $)^{[9]}$. Ces arbres sont des représentations spatiales de concepts mis en jeu dans une situation donnée. Cette approche a connu un grand développement et a donné lieu à des avancées intéressantes dans le domaine de l'apprentissage, notamment avec la cartographie conceptuelle mise au point par Novak et Gowin en $1984^{[10]}$.

\section{Les cartes conceptuelles comme outil d'évaluation}

Une carte conceptuelle est une représentation graphique d'un domaine de la connaissance tel que perçu par un ou plusieurs individus. Cette représentation, évolutive, établit des liens entre des concepts, interconnexions sémantiques, selon des règles plus ou moins formelles ${ }^{[11]}$.

L'idée de carte conceptuelle (concept map) est développée depuis plus d'une trentaine d'années par Novak et ses collègues de la Cornell University. Les travaux de Novak s'inscrivent dans la logique de la conception de l'apprentissage d'Ausubel ${ }^{[12]}$ qui a clairement distingué l'apprentissage machinal de l'apprentissage signifiant, ce dernier prenant en compte les connaissances antérieures de l'apprenant. 
L'élaboration d'une carte conceptuelle permet d'expliciter la pensée d'un individu à travers l'agencement des concepts qu'il met en jeu. Elle oblige l'individu à penser dans différentes directions. Les cartes conceptuelles sont utilisées pour favoriser l'apprentissage mais aussi comme outil d'évaluation $^{[13,14]}$. Elles prennent tout leur sens dans le cadre d'une pédagogie active, visant à créer les conditions favorables à la mobilisation de ressources internes et externes par l'étudiant, en cohérence avec les principes directeurs d'une approche par compétences. En général, trois éléments sont retrouvés dans une carte conceptuelle : a) des concepts encadrés dans une figure géométrique appelés nœuds; b) des liens clairement dénommés entre les concepts ; c) une organisation hiérarchique de l'ensemble des éléments.

La création de cartes conceptuelles par les apprenants constitue une activité favorisant la construction active et réflexive de leurs connaissances. Elle permet un traitement plus profond de l'information, découlant des efforts d'identification des concepts-clés et de leurs relations, la création d'un schéma mental et la réorganisation de l'information. Dans le cadre de l'évaluation d'une compétence, il s'agit pour l'étudiant de constituer une carte qui représente les ressources qu'il a effectivement mobilisées et combinées dans un contexte ou encore, celles qu'il aurait pu mobiliser et combiner.

Il est reconnu que la carte conceptuelle d'un étudiant est unique parce qu'elle représente sa manière de sélectionner des ressources, de les situer hiérarchiquement et de déterminer des liens structuraux. Il est par ailleurs admis que chaque carte devrait partager une base de connaissances ou de ressources communes avec ce que l'enseignant ou le formateur estime comme devant composer cette base. Plusieurs écrits soulignent d'ailleurs l'importance de disposer d'une carte de référence (criterion map), élaborée par un ou plusieurs « experts » dans le domaine afin de jauger le degré de maîtrise des ressources atteint par les étudiants ${ }^{[2]}$.

Cette étude propose d'explorer l'intérêt de la carte conceptuelle pour l'évaluation d'une compétence en soins infirmiers. Il s'agira de repérer à quel point la réalisation d'une carte conceptuelle permet d'identifier les ressources mobilisées et le niveau d'organisation des connaissances pour traiter efficacement une situation.

\section{Matériels et méthodes}

\section{La population ciblée}

L'étude a été réalisée dans un IFSI de la région Bourgogne, au sein d'une promotion de deuxième année, composée de 55 étudiants. Leur première rentrée scolaire a eu lieu en septembre 2009 avec la mise en place du nouveau programme. L'ensemble du référentiel a été présenté aux étudiants et l'ouvrage de référence leur a été donné.

Tous les étudiants de deuxième année ont été évalués et certifiés sur la compétence 3 du référentiel de formation : « accompagner une personne dans la réalisation de ses soins quotidiens ». La proposition de participer à cette étude, avec l'aval du directeur de l'IFSI, a été faite à l'ensemble de ces 55 étudiants de deuxième année et 20 étudiants se sont portés volontaires pour ce travail, soit $36,7 \%$. Les raisons pour lesquelles les autres étudiants n'ont pas participé n'ont pas été explorées.

\section{Le déroulement et les outils de l'étude}

L'étude s'est déroulée de janvier à mai 2011. Dans un premier temps, il a été demandé à trois cadres de santé et formateurs, exerçant dans des établissements différents et utilisant les cartes conceptuelles dans leurs pratiques professionnelles, d'élaborer une carte conceptuelle sur « les ressources pouvant être mobilisées et à combiner par un étudiant en soins infirmiers de deuxième année pour accompagner une personne âgée en institution gériatrique dans la réalisation de ses soins quotidiens ». La consigne leur a été communiquée par oral et par écrit en leur rappelant la définition de référence de la compétence ainsi que ce qui est entendu comme types de ressources contributives à la mise en œuvre de cette compétence. À partir de leurs travaux, nous avons construit une carte de 
référence en nous appuyant sur les propositions communes à deux ou trois des experts. Il en résulte une représentation qui, dans un but didactique, permet de distinguer les deux types de ressources mobilisées lors de la mise en œuvre de la compétence décrites dans la littérature : des ressources internes et externes. L'ensemble de ces ressources constitue les connaissances propres de la personne, encodées dans sa mémoire à long terme. Cette carte leur a été soumise pour validation (figure 1).

Dans un deuxième temps, les étudiants ont été sollicités pour réaliser leur carte conceptuelle. $\mathrm{Au}$ cours de leur formation, les étudiants doivent effectuer au moins un stage dans chacun des quatre contextes suivants : soins de courte durée, soins en santé mentale et en psychiatrie, soins de longue durée/ soins de suite et de réadaptation et soins individuels ou collectifs sur des lieux de vie. Dans cet institut de formation, les stages en soins de longue durée ou à orientation gériatrique sont planifiés en semestre 1 ou 2 (soit cinq ou dix semaines de stage). Tous les étudiants de l'échantillon ont effectué au moins cinq semaines de stage en gériatrie depuis le début de leur formation. Par ailleurs, ils ont suivi au cours du premier semestre deux unités d'enseignement contributives à la compétence 3 (infectiologie hygiène, soins de confort et de bien-être). C'est pourquoi la situation choisie, pour respecter le caractère contextuel de la compétence, a concerné la population âgée en institution, situation rencontrée par tous les étudiants de l'échantillon.

Les objectifs de la recherche leur ont été présentés le 24 février 2011 ainsi qu'une clarification des concepts. Ces étudiants ont bénéficié d'une formation à l'élaboration d'une carte conceptuelle de deux heures. Un exercice au tableau leur a été proposé à partir d'un concept simple. Le logiciel CmapTools ${ }^{[15]}$ leur a été présenté et a pu être installé sur les 10 ordinateurs mis à leur disposition au centre de documentation.

Les consignes du travail leur ont été communiquées au terme de l'atelier et un document les rappelant leur a été donné afin qu'ils puissent en conserver une trace écrite : «élaborer une carte conceptuelle déterminant les ressources que vous avez mobilisées et combinées pour déployer la compétence 3 en situation « Accompagner une personne âgée en institution dans la réalisation de ses soins quotidiens » ».

\section{L'analyse des résultats}

L'analyse des résultats a été réalisée en deux temps au regard de la carte de référence.

Le premier temps a consisté à analyser l'ensemble des cartes produites par les étudiants afin d'identifier : le nombre d'étudiants ayant évoqué les ressources au regard de celles estimées mobilisables dans la situation par des étudiants de deuxième année selon les experts ; les ressources qui ne sont pas évoquées par les étudiants ; l'occurrence de nouvelles propositions (deux concepts reliés entre eux) qui n'apparaîtraient pas dans la carte de référence.

Puis dans un deuxième temps, pour chaque étudiant ont été relevées : la fréquence des ressources internes et externes au regard des ressources attendues dans la carte de référence; la structuration hiérarchique des cartes conceptuelles des étudiants c'est-àdire les différents niveaux retrouvés en fonction des deux types de ressources (internes et externe); l'occurrence des nouveaux concepts qui n'apparaîtraient pas dans la carte de référence ; les propositions erronées ou non valides.

La structuration hiérarchique de la carte de référence se présente en cinq niveaux. Autour du concept central, correspondant aux ressources mobilisées pour la compétence évaluée, nous retrouvons deux concepts globaux - ou de superordination - ${ }^{[12]}$ de niveau 1 (les ressources externes et les ressources internes), qui se déclinent en onze concepts plus spécifiques - dits de subordination - de niveau 2 (les connaissances, les capacités de l'étudiant, les qualités etc.). Ces concepts sont explicités aux niveaux 3 et 4 (par exemple : respect (niveau 3) de la personne âgée, du cadre, des règles (niveau 4)). Le cinquième niveau correspond à des exemples permettant d'illustrer les concepts.

\section{Résultats}

Tous les étudiants volontaires $(n=20)$ ont réalisé une carte conceptuelle, soit un taux de réponse de $100 \%$. 


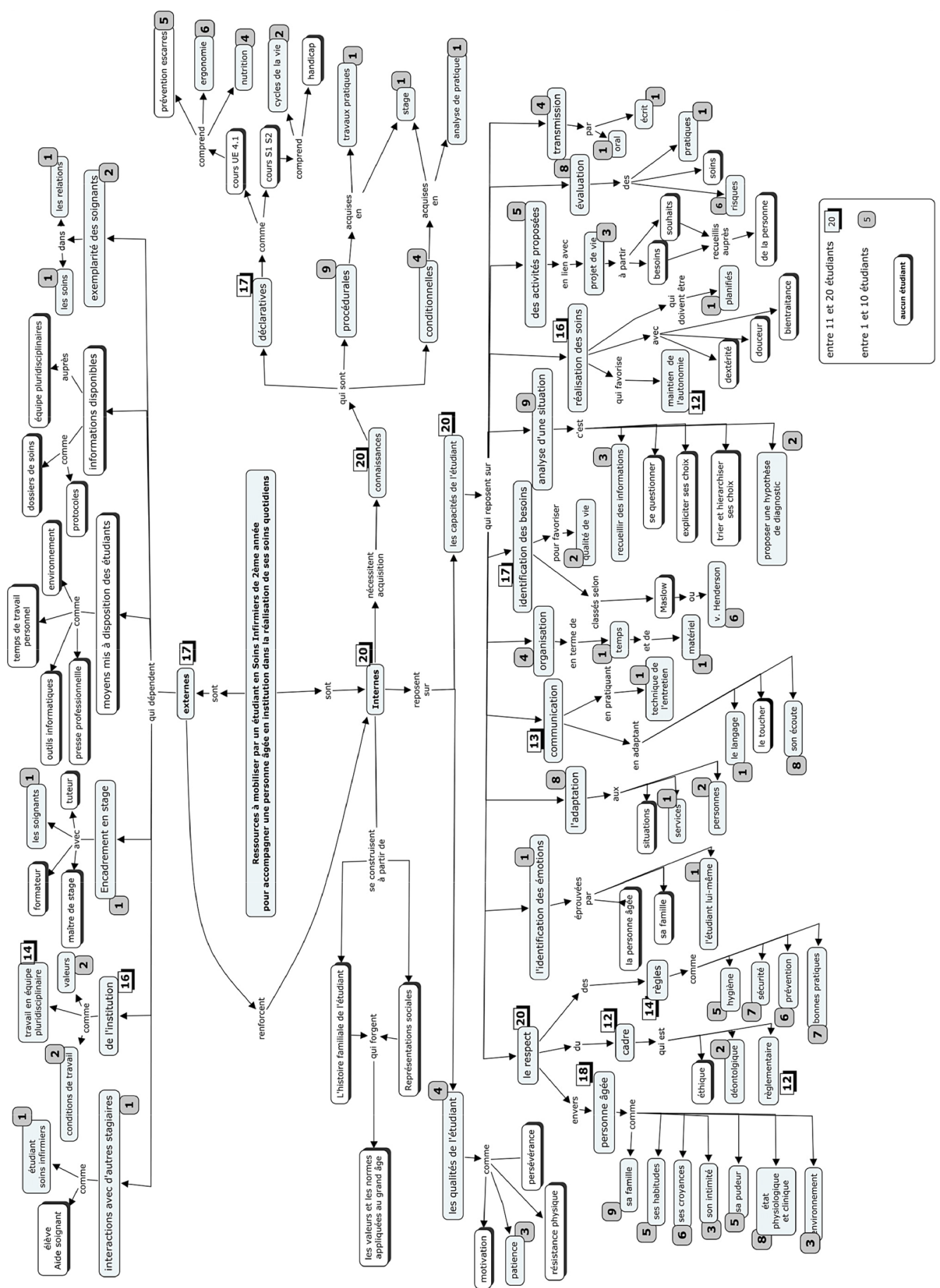

Légende : sont différenciés les concepts retrouvés dans les cartes de plus de la moitié des étudiants, ceux retrouvés seulement dans les cartes de la moitié ou moins des étudiants, et les concepts jamais retrouvés.

Fig. 1. Carte conceptuelle de référence incluant la fréquence des ressources retrouvées dans les cartes conceptuelles des étudiants. 
Seules deux cartes ont été réalisées manuellement. Tous les étudiants ont réalisé cette première carte conceptuelle sans jamais avoir auparavant utilisé le logiciel CmapTools. Le temps moyen de cette charge de travail a été estimé par les étudiants à trois heures et demi (écarts : 1 h30 à 7 h).

La consigne, même si elle a fait l'objet d'une présentation orale et d'un support écrit, a été soumise à interprétation. Les concepts centraux retrouvés, pour lesquelles les ressources ont été déclinées, étaient : « accompagner une personne âgée en institution dans la réalisation de ses soins quotidiens » $(n=13)$, « accompagner une personne dans la réalisation de ses soins quotidiens » $(n=6)$ et « soins d'hygiène et de confort $\gg(n=1)$.

La famille de situations dans laquelle se situait la compétence concernait les personnes âgées en institution. Les soignants ont l'habitude de parler de personnes âgées ou de résidents. Les étudiants font allusion à une personne $(n=6)$, une personne âgée $(n=3)$, un patient $(n=7)$, un résident $(n=2)$ et deux étudiants n'ont donné aucune précision.

Le nombre d'étudiants ayant évoqué chacune des ressources de la carte de référence

Pour faciliter la lecture des résultats, les ressources identifiées par les étudiants ont été intégrées à la carte de référence, mettant en évidence les données attendues non retrouvées (figure 1). Le nombre d'étudiants ayant évoqué chaque concept a été reporté dans la figure 1 avec un code en fonction de leur fréquence.

Les ressources internes sont plus souvent intégrées $(n=20)$ que les ressources externes $(n=17)$ dans le développement de la compétence. Parmi les ressources internes, les étudiants ciblent de façon prioritaire les connaissances $(n=20)$ ainsi que les capacités $(n=20)$. En ce qui concerne les connaissances, plus de trois quarts des étudiants évoquent les connaissances déclaratives qu'ils ont appelées théoriques ou encore «savoir» et la moitié du panel évoque les connaissances procédurales nommées « savoir faire ».
Les capacités les plus retrouvées sont le respect de la personne (âgée, patient, résident) $(n=18)$ et l'identification des besoins de la personne $(n=17)$. Pour trois quarts des étudiants, la réalisation des soins est une ressource et pour plus de la moitié la communication en est une également.

En ce qui concerne l'analyse de situation, elle est perçue comme constitutive de la compétence par moins de la moitié des étudiants $(n=9)$; il en est de même pour les capacités d'adaptation $(n=8)$ et d'évaluation $(n=8)$. La place des émotions, comme caractéristique de l'individu soignant ou soigné, n'a pas été reconnue par les étudiants $(n=1)$.

Certains concepts sont particulièrement développés, avec de nombreux exemples venant les enrichir. Les concepts relatifs au respect du cadre et des règles sont largement détaillés. En ce qui concerne le respect de la personne âgée, la place de la famille $(n=9)$ a été intégrée par les étudiants. Il en est de même pour la prise en compte de l'état physiologique et clinique de la personne $(n=8)$. Le respect du cadre règlementaire $(n=12)$ semble être une préoccupation des étudiants. Ils font figurer l'écoute $(n=8)$ comme principal élément de la communication. L'objectif des soins, en l'occurrence le maintien de l'autonomie de la personne, est mentionné par 12 étudiants.

En ce qui concerne les qualités de l'étudiant, elles n'ont été mentionnées que par à peine un quart du panel et l'impact de l'entourage, des valeurs, des normes véhiculées par la société au regard du grand âge n'a pas été mentionné.

Quant aux ressources externes évoquées par 17 étudiants, seule l'institution $(n=16)$ et plus particulièrement le travail en équipe pluridisciplinaire apparaissent nettement $(n=14)$. Sinon, la grande majorité des ressources externes n'a pas été nommée par les répondants.

Les propositions des étudiants hors la carte de référence

Les propositions des étudiants ont été identifiées afin d'avoir une meilleure représentation de leurs 
conceptions. Tous les étudiants sauf un font des propositions enrichissant la carte de référence. Les étudiants ont particulièrement enrichi la réalisation des soins et les activités.

Ils rattachent la compétence au rôle propre de l'infirmière $(n=4)$ développé en collaboration avec les aides soignantes $(n=4)$. Deux étudiants s'appuient sur les ressources de la personne pour maintenir l'autonomie, ils évoquent également la restauration de l'autonomie. Dans leurs propositions, les étudiants précisent le rôle des soins, ils citent le rôle préventif $(n=1)$, éducatif $(n=2)$, de nursing $(n=2)$, palliatif $(n=2)$, d'accompagnement $(n=3)$. Les étudiants $(n=5)$ parlent de « relationnel », de soins relationnels et de relation d'aide $(n=4)$. Nous avons respecté leur classification mais il ne nous a pas semblé simple de déterminer ce qui était de l'ordre de la communication dans le soin ou de la relation thérapeutique. Deux étudiants inscrivent la réalisation des soins dans un projet de soins individualisé, soins qui doivent être consentis pour trois d'entre eux. Quant au projet de vie, trois étudiants le perçoivent comme permettant à la personne âgée de s'intégrer, de se sentir bien $(n=2)$ et de s'épanouir $(n=1)$.

Trois étudiants évoquent le contexte économique pour la réalisation des soins et des activités soit par la qualité à être économe $(n=1)$ ou par la nécessité de moyens et d'un budget $(n=2)$. La personne âgée est reconnue avec son histoire de vie $(n=4)$, ses goûts $(n=2)$, ses souhaits $(n=7)$. Sa prise en charge repose sur des valeurs professionnelles et éthiques (confort $n=7$, dignité $n=2$ ).

$\mathrm{Si}$, au regard de la carte de référence, les qualités de l'étudiant apparaissaient peu, nous constatons qu'ils sont force de propositions et qu'ils apportent des qualités supplémentaires par rapport à celles identifiées par les cadres de santé : auto gestion, auto évaluation, capacité à réagir, réflexion, disponibilité, ponctualité, observation et plus particulièrement l'empathie citée par quatre étudiants. L'histoire familiale n'a pas été retrouvée mais on note cependant que deux étudiants identifient comme ressource, la connaissance qu'ils ont d'eux-mêmes, leur propre expérience et ce que peuvent leur apporter les personnes âgées. Enfin, ils déclinent les connaissances procédurales comme savoir faire, savoir agir, savoir être et savoir entendre.

\section{Éude analytique des résultats par carte}

Le tableau I présente le total des ressources externes et internes retrouvées dans la carte de chaque étudiant. Un seul étudiant a fait des propositions erronées sur la référence du décret professionnel (1993 au lieu de 2002), sur la qualification des agents de service hospitalier et sur sa conception de la compétence qu'il voit davantage comme une capacité ou une qualité.

Nous constatons que 10 étudiants identifient entre 20 et $30 \%$ des ressources internes de la carte de référence et que parmi eux seuls quatre étudiants évoquent plus de $50 \%$ des concepts de subordination de niveau 2 de ces mêmes ressources. Pour les six autres étudiants (cartes $\mathrm{n}^{\circ} 7,9,15,16,17$ ), les ressources ont été détaillées sans que les principaux concepts soient dégagés.

La carte $n^{\circ} 14$ ne fait apparaître que huit ressources internes, qui correspondent respectivement à huit concepts de subordination.

Comparativement, un seul étudiant identifie $30 \%$ des ressources externes soit sept parmi les 23 de la carte de référence. Les ressources externes ne semblent pas être constitutives du développement de la compétence pour la grande majorité des apprenants.

L'analyse des cartes conceptuelles révèle des niveaux d'abstraction différents si l'on considère le nombre de concepts de subordination de niveau 2 et les ressources qui y sont associées. Certains étudiants s'intéressent à des éléments plutôt concrets. Par exemple dans la carte ${ }^{\circ} 9$, l'étudiant décline l'environnement de la personne comme étant une chambre ou un lieu de vie qui comprend une sonnette, un téléphone, une alarme incendie, un lit, du mobilier de rangement, etc. Seuls quatre étudiants (cartes $\mathrm{n}^{\circ} 4,5$, 10 et 12) identifient plus de $50 \%$ des concepts de subordination de niveau 2 des ressources internes avec un total de ressources supérieur à $23 \%$. Un étudiant (carte $\mathrm{n}^{\circ} 14$ ) identifie huit ressources internes, toutes correspondant à des concepts de surbordination de niveau 2. 
Tableau I. Etude analytique des cartes conceptuelles des étudiants $(n=20)$ par rapport à la carte de référence.

\begin{tabular}{|c|c|c|c|c|c|c|c|c|c|c|}
\hline \multirow{3}{*}{$\begin{array}{l}\text { Cartes des } \\
\text { étudiants } \\
\text { C. } \text { n }^{\circ} 1\end{array}$} & \multicolumn{4}{|c|}{ Ressources internes (RI) } & \multicolumn{4}{|c|}{ Ressources externes (RE) } & \multicolumn{2}{|c|}{ Concepts proposés } \\
\hline & \multicolumn{2}{|c|}{$\begin{array}{c}\text { Total retrouvé } \\
\text { (RI de la carte } \\
\text { de référence }=81 \text { ) }\end{array}$} & \multicolumn{2}{|c|}{$\begin{array}{c}\text { Concepts de } \\
\text { subordination de } \\
\text { niveau } 3(\text { carte } \\
\text { de référence }=19)\end{array}$} & \multicolumn{2}{|c|}{$\begin{array}{c}\text { Total retrouvé } \\
(\mathrm{RE} \text { de la carte } \\
\text { de référence }=23)\end{array}$} & \multicolumn{2}{|c|}{$\begin{array}{c}\text { Concepts de } \\
\text { subordination de } \\
\text { niveau } 2(\text { carte de } \\
\text { référence }=6)\end{array}$} & \multirow{2}{*}{\begin{tabular}{|c|} 
pertinents \\
11
\end{tabular}} & \multirow{2}{*}{ 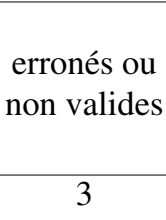 } \\
\hline & 15 & $18,50 \%$ & 6 & $31,50 \%$ & 7 & $30,00 \%$ & 3 & $50,00 \%$ & & \\
\hline C. $n^{\circ} 2$ & 12 & $15,00 \%$ & 5 & $26,00 \%$ & 3 & $13,00 \%$ & 2 & $33,00 \%$ & 3 & 0 \\
\hline C. $n^{\circ} 3$ & 14 & $17,00 \%$ & 6 & $31,50 \%$ & 2 & $8,50 \%$ & 1 & $16,50 \%$ & 8 & 0 \\
\hline C. $n^{\circ} 4$ & 19 & $23,50 \%$ & 11 & $58,00 \%$ & 2 & $8,50 \%$ & 1 & $16,50 \%$ & 7 & 0 \\
\hline C. $n^{\circ} 5$ & 21 & $26,00 \%$ & 10 & $52,50 \%$ & 0 & 0 & 0 & 0 & 6 & 0 \\
\hline C. $n^{\circ} 6$ & 10 & $12,00 \%$ & 5 & $26,00 \%$ & 2 & $8,50 \%$ & 1 & $16,50 \%$ & 4 & 0 \\
\hline C. $n^{\circ} 7$ & 18 & $22,00 \%$ & 7 & $37,00 \%$ & 2 & $8,50 \%$ & 1 & $16,50 \%$ & 8 & 0 \\
\hline C. $n^{\circ} 8$ & 14 & $17,00 \%$ & 5 & $26,00 \%$ & 2 & $8,50 \%$ & 1 & $16,50 \%$ & 4 & 0 \\
\hline C. $n^{\circ} 9$ & 20 & $24,50 \%$ & 9 & $47,00 \%$ & 3 & $13,00 \%$ & 1 & $16,50 \%$ & 4 & 0 \\
\hline C.n ${ }^{\circ} 10$ & 23 & $28,00 \%$ & 12 & $63,00 \%$ & 2 & $8,50 \%$ & 1 & $16,50 \%$ & 11 & 0 \\
\hline C. $n^{\circ} 11$ & 21 & $26,00 \%$ & 8 & $42,00 \%$ & 3 & $13,00 \%$ & 1 & $16,50 \%$ & 5 & 0 \\
\hline C.n ${ }^{\circ} 12$ & 24 & $29,50 \%$ & 11 & $58,00 \%$ & 2 & $8,50 \%$ & 1 & $16,50 \%$ & 4 & 0 \\
\hline C.n ${ }^{\circ} 13$ & 11 & $13,50 \%$ & 4 & $21,00 \%$ & 2 & $8,50 \%$ & 1 & $16,50 \%$ & 5 & 0 \\
\hline C.n ${ }^{\circ} 14$ & 8 & $10,00 \%$ & 8 & $42,00 \%$ & 2 & $8,50 \%$ & 1 & $16,50 \%$ & 6 & 0 \\
\hline C.n ${ }^{\circ} 15$ & 18 & $22,00 \%$ & 7 & $37,00 \%$ & 0 & 0 & 0 & 0 & 0 & 0 \\
\hline C.n ${ }^{\circ} 16$ & 17 & $21,00 \%$ & 6 & $31,50 \%$ & 0 & 0 & 0 & 0 & 1 & 0 \\
\hline C.n ${ }^{\circ} 17$ & 18 & $22,00 \%$ & 7 & $37,00 \%$ & 2 & $8,50 \%$ & 1 & $16,50 \%$ & 1 & 0 \\
\hline C.n ${ }^{\circ} 18$ & 8 & $10,00 \%$ & 4 & $21,00 \%$ & 2 & $8,50 \%$ & 1 & $16,50 \%$ & 4 & 0 \\
\hline C.n ${ }^{\circ} 19$ & 12 & $15,00 \%$ & 4 & $21,00 \%$ & 2 & $8,50 \%$ & 1 & $16,50 \%$ & 4 & 0 \\
\hline C.n ${ }^{\circ} 20$ & 13 & $16,00 \%$ & 6 & $31,50 \%$ & 2 & $8,50 \%$ & 1 & $16,50 \%$ & 1 & \\
\hline
\end{tabular}

Légende: Pour chaque ressource et concept de subordination retrouvés, un nombre total est présenté ainsi que le pourcentage correspondant aux éléments de la carte de référence. Les cellules qui apparaissent surlignées correspondent au \% maximum des ressources retrouvées

\section{Discussion et perspectives}

Cette première étude visant à explorer l'intérêt de la carte conceptuelle pour l'évaluation d'une compétence en soins infirmiers permet de dégager les tendances de l'outil à mettre en évidence les ressources mobilisées par les étudiants selon certaines conditions d'utilisation. L'étude comporte néanmoins quelques limites qui seront discutées.
Limites de l'étude

Le nombre restreint d'étudiants (20 au total) et le caractère contextuel (un seul IFSI de petite taille) ne permettent pas de généraliser les résultats. Par ailleurs, la représentativité de l'échantillon ne peut être affirmée, les cartes conceptuelles réalisées par les étudiants ayant été anonymes. Une description plus précise des caractéristiques des étudiants ayant 


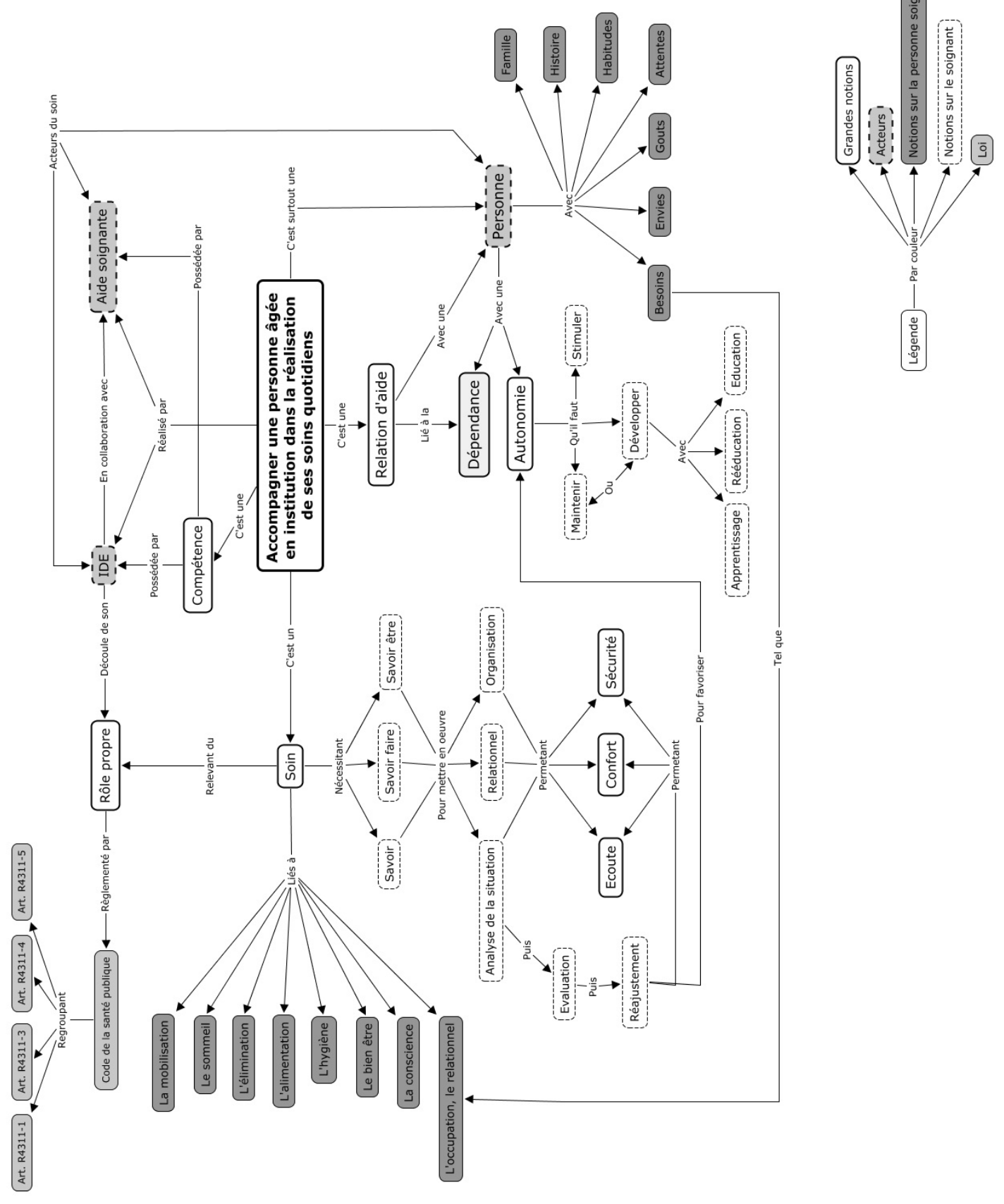

Légende : Outre la présentation des ressources internes et externes mobilisées par l'étudiant, celui-ci a souhaité faire ressortir plus particulièrement par des symboles différents les éléments suivants : ce qu'il considère comme des grandes notions, les concepts qui désignent les acteurs, les notions spécifiques à la personne soignée, et aux soignants, les concepts en lien avec les lois.

Fig. 2. Exemple de carte conceptuelle élaborée par un étudiant. $\left(N^{\circ} 4\right)$. 
participé à l'étude aurait permis d'apprécier les origines des participants, en particulier celles relatives à leur cursus éducatif. En effet, les promotions d'étudiants des IFSI sont le plus souvent très hétérogènes, certains étant déjà des professionnels, d'autres ayant pu suivre ou non un cursus universitaire après le baccalauréat (diplôme de fin d'études secondaires en France) avant d'intégrer l'IFSI.

Les cartes produites par les étudiants ont pu être analysées au regard d'une carte de référence. Les limites concernant ce type d'analyse ont été décrites dans la littérature. En effet, il reste difficile d'établir une norme, de construire un profil de compétences standardisées $^{[16]}$. Augmenter le nombre d'experts permettrait d'élargir l'appréciation de la compétence. Nous avons pallié en partie cette limite en analysant également les éléments nouveaux apportés par les étudiants. Par ailleurs, sans phase d'explicitation de leur carte par les étudiants, nous avons été contraints à l'inférence, processus à travers lequel l'évaluateur, le chercheur, tente de produire du sens en donnant une signification aux informations qu'il recueille ${ }^{[7]}$. Certaines ressources identifiées par les étudiants ont été interprétées, comme par exemple «s'adapter à son mode de vie» en «respecter son mode de vie». Quand nous avons trouvé « comprend » $\rightarrow$ « règles d'ergonomie », nous avons dû opter avec difficulté entre la ressource concernant les connaissances déclaratives et la ressource concernant le respect des règles. Une double analyse aurait permis de limiter les éventuelles erreurs d'interprétation.

Enfin, l'analyse produite dans cette étude peut être considérée comme incomplète, n'ayant pas pris en compte de manière approfondie l'organisation des cartes conceptuelles. Si la structure hiérarchique des cartes a été en partie analysée au travers des différents niveaux de concepts de subordination (cinq niveaux attendus), les liens interconceptuels auraient pu faire l'objet d'une analyse complémentaire permettant d'apprécier plus précisément la structure en réseau des cartes conceptuelles, qualité d'organisation nécessaire à l'expertise ${ }^{[17]}$.

Enfin, en termes de limite, la carte conceptuelle ne permet pas d'évaluer une compétence mais bien une représentation graphique des ressources mobilisées lors de la mise en œuvre d'une compétence dans une situation donnée. L'identification des ressources par les étudiants n'est pas garante de sa capacité à les utiliser à bon escient en contexte, on ne pourra donc pas parler d'évaluation authentique ${ }^{[2]}$. Il conviendra donc de s'assurer que la situation réelle dans laquelle est placé l'étudiant, l'incite à la mobilisation des ressources pour démontrer la compétence.

\section{Intérêts de la carte conceptuelle}

Les cartes conceptuelles apportent des informations précieuses aux formateurs leur permettant d'améliorer leurs enseignements. L'analyse des ressources présentes dans la carte et de leur niveau de développement est un indicateur du processus de développement de la compétence chez l'étudiant. L'absence de développement de certaines ressources interroge sur les contenus et les stratégies d'enseignement.

L'étude montre qu'il est nécessaire de développer des stratégies pédagogiques pour faire émerger les ressources externes par les étudiants. Par ailleurs, concernant les ressources internes, très peu d'étudiants ont développé les capacités qui reposent sur les activités proposées à la personne âgée $(n=5)$ entendues comme loisirs ou activités occupationnelles et le projet de vie $(n=3)$. Ce sont pourtant des particularités spécifiques aux établissements d'hébergement gériatrique. Il est possible qu' au stade de leur formation la notion de soin soit perçue davantage comme soins techniques ou de nursing $(n=16)$, alors que dans cette situation les soins sont considérés comme «action ou ensemble d'actions qu'une personne décide et accomplit pour elle-même en vue de maintenir, protéger, restaurer et promouvoir la santé » (Dictionnaire des soins infirmiers, Masson 2005). Le formateur doit donc veiller à ce que la santé de la personne âgée ne se réduise pas aux besoins d'hygiène, d'alimentation, de mobilisation, d'élimination des personnes. Les valeurs et les normes appliquées au grand âge ne font pas parties des ressources des étudiants. Elles ne sont ni rattachées à leur histoire familiale, ni aux représentations sociales. Un seul étudiant signale la place de son expérience personnelle dans le développement de la compétence. Pourtant, 
comme l'a étudiée la sociologie de l'éducation, le contexte a des effets sur les performances cognitives des individus. Une hypothèse d'explication pourrait être que les étudiants n'ont pas conscience de l'habitus, c'est-à-dire de la façon dont les structures sociales influencent leurs manières d'être, de penser, de percevoir et d'agir dans le monde. C'est l'habitus qui explique la reproduction des rapports sociaux, à l'insu des acteurs eux-mêmes ${ }^{[18]}$. Le formateur pourrait donc s'appuyer sur la carte conceptuelle de l'étudiant révélant sa représentation du soin chez la personne âgée pour l'aider à prendre conscience de cet habitus.

La notion d'éthique n'est quasiment pas retrouvée, ce qui peut s'expliquer du fait que le sujet est davantage traité au semestre 4 de la formation et qu' au moment de l'étude les étudiants l'ont peu abordé. De même le concept de la communication, pourtant traitée aux semestres 2 et 3 , reste peu développé. Le formateur pourrait davantage travailler avec les étudiants la notion d'écoute (citée par huit étudiants seulement) ainsi que les autres modes de communication non retrouvés dans les cartes (communication non verbale, la qualité de l'expression du toucher). Quelques étudiants ont évoqué dans leur carte conceptuelle la notion de relation d'aide. Cette notion, non initialement intégrée à la carte de référence car abordée à la fin du semestre 3 (période de l'étude), pourrait être ajoutée. La relation d'aide vise des objectifs précis et déterminés avec la personne ${ }^{[19]}$. Elle nécessite de reconnaître le ressenti de la personne soignée, ses affects, ses émotions. Elle passe par la connaissance de soi et du patient. Or, seul un étudiant cite les émotions éprouvées par lui-même dans sa carte. Pourtant, sous la contrainte sociale, les émotions sont contenues, canalisées, maitrisées. Goleman ${ }^{[20]}$ précise que chaque émotion nous prépare à agir d'une certaine manière et que dans notre répertoire d'émotions, chacune joue un rôle spécifique. Nous trouverions intéressant de développer cette approche dans la formation des futurs soignants pour leur permettre de faire un diagnostic d'une situation affective.

Dans les cartes conceptuelles de quatre étudiants, la compétence est reliée à leur rôle propre en collaboration avec les aides-soignantes. Nous pouvons supposer que leur identité professionnelle, en considérant la dimension prescriptive et la dimension d'autonomie, n'est pas encore développée au stade de leur formation ${ }^{[21]}$. La finalité de la formation est bien de permettre à l'infirmier d'assumer chacun de ses rôles : rôle prescrit, rôle propre. Ce dernier constat montre bien comment la carte conceptuelle rend compte du niveau de développement de la compétence chez l'étudiant au regard des ressources mobilisées au moment de l'évaluation.

L'analyse des cartes conceptuelles révèle des niveaux d'abstraction différents ${ }^{[22]}$ si l'on considère le nombre de concepts de subordination de niveau 2 et les ressources qui y sont associées. Certains étudiants s'intéressent à des éléments plutôt concrets. Par exemple, le niveau de détail explicité dans la carte $\mathrm{n}^{\circ} 9$ évoque la théorie de B-M Barth ${ }^{[22]}$ selon laquelle notre conception de la réalité est liée à notre capacité à la décrire. Quand le mot renvoie à une situation précise, il sert de « savoir provisoire » qui, au fil du temps et des expériences, va s'enrichir. Certains étudiants expriment des concepts plus abstraits en ayant les capacités de les décrire, comme dans les cartes $n^{\circ} 4$, 5,10 et 12 . Cependant, nous nous interrogeons sur le cheminement de l'étudiant ayant élaboré la carte $\mathrm{n}^{\circ} 14$ (qui contient huit ressources internes retrouvées, toutes correspondant à des concepts de surbordination niveau 2) : ces résultats révèlent-ils un niveau d'abstraction élevé ou bien une incompréhension des concepts évoqués par manque d'illustration de ces concepts?

\section{Perspectives d'utilisation}

L'ensemble des analyses précédentes permet d'envisager les objectifs poursuivis par l'élaboration d'une carte conceptuelle pour documenter la compétence. Elle pourrait être une première étape pour l'identification des ressources associées au développement d'une compétence selon certaines conditions. L'étude signale que la définition de la compétence doit être clarifiée au niveau de l'équipe pédagogique mais également auprès des étudiants si l'on souhaite aborder son évaluation. La réalisation de la carte conceptuelle a permis aux étudiants de concrétiser le concept de compétence. Par ailleurs, les apprenants semblent s'appuyer essentiellement sur les ressources internes 
pour développer la compétence. Une phase d'explicitation et de rétro-action à partir de la carte pourrait permettre d'enrichir encore les cartes des étudiants et d'apprécier si les étudiants se réfèrent uniquement à ce type de ressources par choix délibéré ou s'ils n'ont pas conscience que les ressources externes contribuent également au développement de la compétence ${ }^{[6]}$, ce qui pourrait expliquer le faible nombre de ressources externes retrouvées. Ces constats soulignent l'intérêt qu'il y a à travailler auprès des étudiants leurs représentations du concept de compétence en préalable au développement de celle-ci. C'est à l'enseignant d'aider les étudiants à prendre conscience des différentes ressources à mobiliser. Développer la compétence nécessite un besoin d'apprentissage en incluant l'explicitation de ses composantes, de ses ressources ${ }^{[2,3]}$

De même, une phase d'explicitation et de rétro information pourrait potentialiser les effets de la carte conceptuelle sur l'apprentissage des étudiants. Les bénéfices de la rétro-information associée à la carte conceptuelle ont été montrés par plusieurs auteurs ${ }^{\text {[23] }}$. Par exemple, très peu d'erreurs ont été relevées dans les cartes produites par les étudiants. Une rétro-information auprès des étudiants sur leur carte pourrait avoir un effet positif sur leur sentiment d'efficacité personnelle et sur leur motivation à apprendre par la valorisation qu'elle permet ${ }^{[3]}$. Cette phase pourrait être réalisée individuellement ou en groupe. Ces pratiques s'inscrivent dans un processus d'accompagnement, tourné vers l'apprenant, qui va l'informer sur la qualité de sa production et lui permettre de la moduler, la modifier.

Lors de ce travail, les étudiants ont exprimé librement et ouvertement les ressources sur lesquelles ils ont pris appui. Ils se sont investis dans cette expérimentation sans doute parce qu'ils l'ont vécu davantage comme une évaluation formative plutôt qu'un contrôle aboutissant à une note. Cela renvoie à la perception qu'a l'étudiant de l'évaluation et de sa fonction. Selon Roegiers ${ }^{[7]}$ l'étudiant prendra moins de risque dans une évaluation donnant lieu à une note alors qu'il se révélera davantage tel qu'il est quand il sait qu'il ne sera pas sanctionné. Il nous semble donc important d'envisager l'utilisation de la carte conceptuelle comme un outil d'évaluation formative ${ }^{[24]}$.
Reste à travailler le caractère transférable des ressources dans d'autres situations. Il est important de proposer aux étudiants d'appréhender la compétence « accompagner une personne dans la réalisation de ses soins quotidiens » dans des situations variées pour favoriser le transfert, phénomène qui n'est ni spontané ni automatique. Ce pourrait être auprès d'une personne âgée en chirurgie, après une intervention, suite à une fracture du col du fémur, auprès d'un homme ayant subi une amputation de la jambe suite à un accident du travail, auprès d'un jeune schizophrène suivi en hôpital de jour. Nous pourrions identifier les ressources invariantes de la compétence et celles spécifiques à la situation, au contexte.

D'autres perspectives d'utilisation de la carte conceptuelle comme soutien à l'apprentissage sont envisagées. En particulier, il pourrait y avoir un intérêt à faire élaborer par les étudiants le même type de carte conceptuelle avant leur départ en stage. Cette carte conceptuelle deviendrait la propre grille d'évaluation de l'étudiant en stage par rapport à la compétence, charge à lui de la faire évoluer en fonction de ses apprentissages.

\section{Conclusion}

Même si la compétence se développe dans l'action, il est important de pouvoir en formaliser les ressources afin de se mettre d'accord sur les éléments auxquels il convient de porter son attention. Les résultats de cette étude exploratoire font apparaître que les étudiants identifient des ressources mobilisées dans le développement d'une compétence, en référence à une carte réalisée par des experts. En ce sens, la carte conceptuelle a un intérêt pour contribuer à l'évaluation formative d'une compétence. La carte conceptuelle témoigne non pas d'un résultat mais d'une compétence en construction, d'un processus. Elle permet une évaluation des apprentissages et des limites à faire reculer grâce à une rétro information sur la carte conceptuelle et un ajustement des enseignements. Des améliorations doivent être apportées à la carte de référence, tant au niveau des ressources qu' au niveau de sa structure, en particulier par rapport à son caractère intégrateur. 
Les potentialités de la carte conceptuelle sur l'apprentissage de l'étudiant seront d'autant plus importantes qu'il sera donné aux étudiants de multiples occasions d'en réaliser. Il serait intéressant d'explorer auprès des étudiants impliqués dans l'étude quel a été leur intérêt à réaliser ce travail et ses conséquences.

\section{Contributions}

Cet article a été rédigé par Pascale Chastragnat qui a mené l'étude dans le cadre d'un travail de master en Santé Publique, spécialité «Ingénierie des Formations en Santé » à l'Université Paris 13. Claire Marchand a dirigé ce travail et a contribué à l'amélioration et la révision de l'ensemble du manuscrit.

\section{Annexe n ${ }^{\circ} 1$ \\ Les dix compétences du référentiel de formation Annexe II de l'arrêté du 31 juillet 2009 relatif au diplôme d'État d'infirmier}

1 - Évaluer une situation clinique et établir un diagnostic dans le domaine infirmier.

2 - Concevoir et conduire un projet de soins infirmiers. 3 - Accompagner une personne dans la réalisation de ses soins quotidiens.

4 - Mettre en œuvre des actions à visée diagnostique et thérapeutique.

5 - Initier et mettre en œuvre des soins éducatifs et préventifs.

6 - Communiquer et conduire une relation dans un contexte de soins.

7 - Analyser la qualité des soins et améliorer sa pratique professionnelle.

8 -Rechercher et traiter des données professionnelles et scientifiques.

9 - Organiser et coordonner des interventions soignantes.

10 - Informer et former des professionnels et des personnes en formation.

\section{Références}

1. Burns R. Methods for individualizing instruction. Educational Technology 1971;11:55-6.

2. Tardif J. L'évaluation des compétences. Montréal : Chenelière éducation, 2006.

3. Scallon G. L'évaluation des apprentissages dans une approche par compétences. $2^{\mathrm{e}}$ éd. Bruxelles : Editions De Boeck Université, 2007.

4. Le Boterf G. Construire les compétences individuelles et collectives. ( $5^{\mathrm{e}}$ éd.). Paris : Eyrolles, 2010.

5. Tardif J. Pour un enseignement stratégique. L'apport de la psychologie cognitive. Montréal : Les Éditions Logiques, 1997.

6. Le Boterf G. Repenser la compétence. Paris : Eyrolles, 2008.

7. Roegiers X. L'école et l'évaluation. ( $2^{\mathrm{e}}$ éd.) Bruxelles : De Boeck Université, 2010.

8. Association pour la pensée complexe fondée par Edgar Morin. [On-line] Disponible sur: http:// www.mcxapc.org/static.php?file=apc.htm\&menuID =APC (consulté le 12 septembre 2010).

9. Nicolas MT. Enseigner la médecine : comment apprendre à faire des liens. Mémoire pour le master « didactique et socialisation ». Laboratoire Interdisciplinaire de Recherche en Didactique, Éducation et Formation. Montpellier: Université Montpellier, 2006.

10. Novak JD, Gowin DB. Learning how to learn ( $4^{\mathrm{e}}$ éd.). New York: Cambridge University Press, 1989.

11. Laflamme A. Les cartes conceptuelles : un outil pour soutenir l'acquisition des connaissances. CEFES université de Montréal. 2006. [On-line] Disponible sur : http://www1.sites;fse.ulaval.ca/fichiers/ site_mmottet/.../CmapTools.pdf

12. Ausubel DP. Educational Psychology: A Cognitive View. New York: Holt, Reinhart \& Winston, 1968.

13. Marchand C, d'Ivernois JF. Les cartes conceptuelles dans les formations en santé. Pédagogie Médicale 2004;5:230-40.

14. Marchand C, Gagnayre R. Utilisation de la carte conceptuelle auprès de formateurs en santé pour l'apprentissage de concepts pédagogiques. Pédagogie Médicale 2004;5:13-23.

15. Cmap Tools [On-line] Disponible sur : http:// cmap.ihmc.us 
16. Ruiz-Primp MA. Shavelson R. Problems and issues in the use of concept maps in science assessment. J Res Sci Teach 1996;33:569-600.

17. Bordage G. Lemieux M. Elaborated knowledge: a key to successful diagnostic thinking. Acad Med 1994;69-11:883-5.

18. Bourdieu P, Passeron J-C. La reproduction : éléments pour une théorie du système d'enseignement. Paris : Les éditions de Minuit, 1970.

19. Daydé MC, Lacroix ML, Pascal C. La relation d'aide en soins infirmiers. ( $2^{\mathrm{e}}$ éd.) Paris : Elsevier Masson, 2010.

20. Goleman D. L'intelligence émotionnelle : accepter ses émotions pour développer une intelligence nouvelle. J 'ai Lu. Paris : Robert Laffont, 1997.
21. Marchal A, Psiuk T. Le paradigme de la discipline infirmière en France. Comprendre, pratiquer, enseigner et apprendre. Paris : Editions Seli Arslan, 2002.

22. Barth BM. Le savoir en construction. Former à une pédagogie de la compréhension. ( $3^{\mathrm{e}}$ éd.) Paris : Éditions Retz, 2004.

23. Daley BJ, Torre DM. Concepts maps in médical éducation: an analytic littérature review. Med Educ 2010;44:440-48.

24. Talbot L. L'évaluation formative : comment évaluer pour remédier aux difficultés d'apprentissage. Paris : Armand Colin, 2009.

Correspondance et offprints: Pascale Chastragnat, IFMS, Étienne Villain, 1 avenue Pierre de Coubertin, 89100 Sens, France. Mailto : pascalechastragnat@free.fr 